P207 SYPHILIS: SIGNIFICANT INCREASE IN MEN WHO HAVE SEX WITH MEN (MSM) SINCE NOVEMBER 2013

${ }^{1}$ Nicolas Pinto-Sander*, 'Elaney Youssef, ${ }^{2}$ Marc Tweed, 'Gillian Dean, ${ }^{1}$ Daniel Richardson. ${ }^{1}$ Brighton and Sussex University Hospitals NHS Trust, Brighton, UK; ${ }^{2}$ Terrence Higgins Trust South, Brighton, UK

\subsection{6/sextrans-2015-052126.251}

Background High rates of infectious syphilis have been reported in MSM. Locally 1:10 of the population are estimated MSM and high rates of HIV and sexually transmitted infections are seen.

Methods We identified cases of infectious syphilis in MSM per month from February-2013 to June-2014 attending sexual health services. Age, ethnicity, HIV status and syphilis re-infection were noted. The total number of MSM seen in sexual health was used as a denominator for incidence calculations and rates were compared using Chi-square and Mann-Whitney test.

Results 207 new cases of infectious syphilis were identified over the study period. The median age was 36 years (19-60), 96/207 (46.4\%) were HIV+ and 3/207(1.4\%) had syphilis re-infection. The median incidence of syphilis from February to October 2013 was 8.6/1000 MSM; this increased significantly to 25.9/ 1000 MSM from November-June 2014 [chi-square 67.447 $(\mathrm{p}<0.0001)$. There was no significant difference in the percentage co-infected with HIV between these time points (FebruaryOctober $=57 \%$, November-June $=46 \%$ [Mann-Whitney $=$ $16.5, \mathrm{p}=0.0581)$.

Conclusion We describe a significant increase in the incidence of infectious syphilis in MSM from November 2013. This rise is likely attributable to changes in sexual behaviour among MSM: increased accessibility to sex driven by social media, increased anonymous and group sex and growing use of party drugs. Locally we are working with the Terrence Higgins Trust and public health teams to increase awareness among MSM and primary care.

\section{P208 MEN'S BEHAVIOUR CHANGE FOLLOWING A POSITIVE AND NEGATIVE DIAGNOSIS FOR CHLAMYDIA}

${ }^{1}$ Michelle Stamp ${ }^{1 *}$. 'University of Northumbria at Newcastle, Newcastle Upon Tyne, UK; ${ }^{1}$ The Newcastle Upon Tyne Hospitals Trust, Newcastle Upon Tyne, UK

\section{$10.1136 /$ sextrans-2015-052126.252}

Background/introduction Young men aged 20 to 24 years who are screened through the National Chlamydia Screening Programme have a high rate of infection. The majority of them choose to self-request screening via anonymous postal testing as opposed to seeking alternative health service provision.

Aim(s)/objectives To explore the complex factors involved in men's sexual health decision making following a request for an internet test for chlamydia.

Methods Ten young men who had requested a test for chlamydia via the internet were recruited through the North of Tyne Chlamydia Screening Programme. Data were collected through in-depth interviews, follow-up interviews at 12 months and patients' NHS health records.

Results Decisions about sexual partners and sexual practice were based on men's perceptions and belief about women, categorising them as 'risky' with a sexually transmitted infection or 'clean' with no infection. Factors influencing decisions to seek testing were triggered by unprotected sex with casual partners, strengthened by catalytic influences including media campaigns.
The findings suggest a negative chlamydia test result gave respondents a clean bill of health allowing them to engage in further unprotected sex. A positive diagnosis resulted in shortterm behaviour change and modified sexual practice. After follow up interviews, behaviour change was not maintained and many became re-infected within 6 months.

Discussion/conclusion This has implications for the transmission of chlamydia infection in terms of infection spreading, re-infection of partners and complications to their own health. Further work is required around interventions for chlamydia screening which focus on behaviour change as opposed to screening volume.

\section{P209 'THE GRAND SLAM': SURVEYING THE LOCAL LANDSCAPE OF 'CHEMSEX' DRUG USE AMONGST MSMS IN A COMMUNITY OUTREACH SEXUAL HEALTH CLINIC}

${ }^{1}$ Kevin Turner, ${ }^{1}$ Billy Clarke, ${ }^{1}$ Cecilia Priestley ${ }^{*},{ }^{1}$ Sara Scofield, ${ }^{2}$ Cordelia Chapman. ${ }^{1}$ Dorset County Hospital NHS Foundation Trust, Weymouth, UK; ${ }^{2}$ The Royal Bournemouth and Christchurch Hospitals NHS Trust, Bournemouth, UK

\subsection{6/sextrans-2015-052126.253}

Background 'Chemsex' is the use of recreational drugs before and/or during sex by men who have sex with men (MSM). Concern exists among health professionals about this practice particularly in relation to transmission of HIV and sexually transmitted infections (STIs). There is little data about use of Chemsex outside London.

Aims/objectives To generate a profile of Chemsex use amongst MSM accessing a community outreach sexual health clinic.

Methods In 2014 a local code was introduced to identify reported use of Chemsex. A retrospective case note audit was undertaken of patients identified during 2014.

Results 636 patients attended for STI screening in 2014. Overall $24 \%$ had an STI. There were 46 attendances in 40 MSM where Chemsex was reported. The average age was 35(19-62). 24/40 $(60 \%)$ were single. 39/40(98\%) reported oral sex, 30/40(75\%) insertive and 27/40(68\%) receptive anal sex with only 6(15\%) reporting consistent condom use. Mephedrone (MCAT) was the most commonly used drug, reported by $33 / 40(83 \%)$. Gammabutyrolactone $(\mathrm{G})$ was used by $10 / 40(25 \%)$. Crystal Meth (Meth) was used by $7 / 40(18 \%)$. 9/40(23\%) used MCAT combined with G. 2/40(5\%) used all three drugs. 35/40(88\%) were asymptomatic. 23/40(58\%) had previously had an STI. 6/40(15\%) were HIV positive.16/40(40\%) patients were diagnosed with an STI (13 gonorrhoea (3 dual site site) and 6 chlamydia (all single site) and 3 both chlamydia and gonorrhoea.

Discussion/conclusions Introduction of a local code has given insight into Chemsex use amongst MSM. We have updated the clinic history proforma to identify high risk behaviour, allowing targeted intervention to facilitate positive behavioural change.

\section{P210 UPTAKE AND ACCEPTANCE OF COMBINED HIV POCT AND STI SCREENING FOR MSM IN COMMUNITY SETTINGS DURING NATIONAL HIV TESTING WEEK}

${ }^{1}$ Kevin Turner, ${ }^{1}$ Billy Clarke, ${ }^{1}$ Cecilia Priestley ${ }^{*},{ }^{1}$ Sara Scofield, ${ }^{2}$ Cordelia Chapman. ${ }^{1}$ Dorset County Hospital NHS Foundation Trust, Weymouth, UK; ${ }^{2}$ The Royal Bournemouth and Christchurch Hospitals NHS Trust, Bournemouth, UK

10.1136/sextrans-2015-052126.254 
Background/introduction National HIV Testing Week (NHTW) aims to increase the earlier detection and treatment of HIV by increasing access to testing across community and statutory settings, with a focus on at risk populations including men who have sex with men (MSM). However there are increasing concerns about risky behaviour, including Chemsex, and an increase in other STIs in MSM.

Aims/objectives To review the acceptance by MSM, of full sexually transmitted infection (STI) screening in a community setting, during NHTW.

Methods We promoted NHTW using national and local material, shared across social media platforms aimed at MSM. In addition to HIV point of care testing (POCT) using a 4th generation test, we offered full STI screening (urine and self-taken pharyngeal/rectal swabs for chlamydia and gonorrhoea NAATs and syphilis POCT). Sexual histories were self-completed.

Results 74 patients were screened; 56 identified as MSM; average age $33(17-75)$. Of these only $21(38 \%)$ reported consistent condom use for anal sex. $4(7 \%)$ reported Chemsex, with MCAT the commonest drug. 20(36\%) had a past history of an STI. 42(75\%) underwent full screening, 12(21\%) POCT only (10 HIV and syphilis, $2 \mathrm{HIV}$ ), and 2(4\%) for chlamydia and gonorrhoea only. There were 3 positive diagnoses: $1 \mathrm{HIV}, 1$ pharyngeal gonorrhoea and 1 rectal chlamydia.

Discussion/conclusion NHTW has proven its effectiveness in increasing the uptake of HIV testing in at risk populations; we have shown that offering full sexual health screening as part of NHTW activity, using self-taken history and urine / non-invasive swabs, is acceptable and effective.

\section{P211 MSM THE COST OF HAVING A GOOD TIME? A SURVEY ABOUT SEX, DRUGS AND LOSING CONTROL}

Nicky Dearing ${ }^{*}$, Sarah Flew. Nottingham University Hospitals NHS Trust, Nottingham, UK

\subsection{6/sextrans-2015-052126.255}

Background Men who have sex with men (MSM) suffer substantial health inequalities compared to the rest of the population relating to sexual health, mental health and drug use. Increasing substance misuse in a sexualised context (chemsex) has been linked to risky sexual behaviour and STI acquisition.

Aims Determine the prevalence of chemsex in our local MSM population, and associated risks to sexual health.

Methods Men attending a GU clinic during December 2014, who identified as MSM, were invited to complete an anonymous questionnaire. 53 questionnaires were received.

Results Overall, 53\% reported some form of recreational drug use. $38 \%$ reported having chemsex. Chemsex participants were more likely to use mephodrone and Viagra than ecstasy and cocaine used more frequently by other party drug users. $47 \%$ of MSM surveyed used the internet to meet partners. The number of partners (any kind of sexual contact) was similar for MSM using drugs and those not. Unprotected receptive anal sex, including with a partner of unknown HIV status, was higher for MSM reporting chemsex. Men reporting chemsex were less likely to have an up-to-date HIV test $(40 \%$ untested in previous year). Overall $40 \%$ reported having an STI in the last year (most commonly Gonorrhoea). All those receiving an HIV diagnosis in the last year $(n=3)$ were amongst the chemsex group. $49 \%$ reported a mental health problem, with $60 \%$ of chemsex participants having a history of depression and/or anxiety.
Conclusion Tackling the sexual health inequalities of MSM is complex, with substance misuse, social media, and mental wellbeing having an increasing influence.

\section{\begin{tabular}{|l|l}
\hline P212 DO YOU KNOW WHO THE MALE SEX WORKERS ARE IN \\
\hline
\end{tabular} YOUR COHORT?}

${ }^{1}$ Sarah Stockwell*, ${ }^{1}$ Gillian Dean, ${ }^{2}$ Marc Tweed, ${ }^{2}$ Tom Boyt. ${ }^{1}$ Brighton and Sussex University Hospitals NHS Trust, Brighton, UK; ${ }^{2}$ Terrance Higgins Trust, Brighton, UK

\section{$10.1136 /$ sextrans-2015-052126.256}

Background/introduction Pro(TECT) is a bespoke service for male sex workers (MSW) launched by THT in June 2014. The service provides MSW with point of care HIV tests, STI and blood borne virus screening, as well as motivational interviewing, harm reduction and signposting to local SH services.

Aim(s)/objectives To describe the sexual health of MSW engaging with the local GU clinic.

Methods The notes of the Pro(TECT) clients who attended the local GU clinic were reviewed.

Results 15 MSW aged 20-57 years, attended the Pro(TECT) service from June to December 2014. 87\% (13/15) had ever attended the GU clinic; 10 in the last 12-months. Only 3 revealed they were MSW. 39\% (5/13) were HIV positive, of whom 3 had detectable viral loads (42,000 to 307,000 copies/ $\mathrm{ml})$. CD4 counts ranged from $6-698 \times 10^{6} / 1.85 \%$ were hepatitis B immune; 1 was hepatitis C co-infected (viral load 100, $4492 \mathrm{cp} / \mathrm{ml})$.

33 STI screens were performed in the last 12 -months, with an average of 3 screens/person. 39\% (5/13) had an acute STI: 4 rectal, 3 pharyngeal, 1 urethral gonorrhoea; 2 rectal chlamydia; 1 latent (early/late) syphilis. PEP was used by 2 of the 5 HIV negative MSW a total of 5 times in 12-months.

Discussion/conclusion There is a high burden of STIs in this group and a significant risk of onward transmission. MSW may not disclose their work to health care professionals (HCP), even on direct questioning. Identification of MSW is fundamental in order to reduce risk and minimise harm. Pro(TECT) acts as a unique gateway into mainstream services, including advice to access PEP.

\section{P213 PRO(TECT) SERVICE - ENGAGING WITH MALE SEX WORKERS}

${ }^{1}$ Sarah Stockwell*, 'Gillian Dean, ${ }^{2}$ Mark Tweed, ${ }^{2}$ Tom Boyt. 'Brighton and Sussex University Hospitals NHS Trust, Brighton, UK; ${ }^{2}$ Terrance Higgins Trust, Brighton, UK

\subsection{6/sextrans-2015-052126.257}

Background/introduction Pro(TECT), a bespoke service for male sex workers (MSW), was launched in June 2014 by THT to engage with local high-risk MSM. Motivational interviewing (MI) is used to explore behaviour change; clients are offered a wide range of support to improve sexual health and reduce onward STI/HIV transmission. Analysis of behaviour and service evaluation was performed at 6 months.

Methods All clients (15) completed an online survey regarding sexual practices, drug/alcohol use and experience of the service.

Results In the last 12 months: 70\% reported insertive UAI; 40\% receptive UAI; 36\% diagnosed with an STI; 29\% HIV positive; $21 \%$ injected drugs; $43 \%$ under the influence of drugs/alcohol while selling sex; 33\% self-harmed in last 12 months. 